\title{
Toward a centralized data management center for integrated landslide monitoring in Emilia Romagna Region (Italy)
}

\author{
A. Corsini, F. Bonacini, G. Ciccarese, M. Mulas \& F. Ronchetti \\ Università di Modena and Reggio Emilia, Dipartimento di Scienze Chimiche e Geologiche, Modena, Italy
}

\author{
S. Nanni \\ Lepida S.p.A.-Area Research and Development, Bologna, Italy \\ G. Truffelli, G. Caputo, M. Pizziolo, S. Primerano \& A. Monni \\ Regione Emilia Romagna, Servizio Tecnico Bacino Po-Servizio Tecnico Bacino Reno-Servizio \\ Geologico-Agenzia Regionale di Protezione Civile, Parma and Bologna, Italy
}

\begin{abstract}
In Emilia Romagna Region, slope monitoring systems have become more widely used for hazard and risk management. However, they are generally non-interoperable. Moreover dispersion of monitoring data in several local databases have made data sharing among the involved institutional actors quite laborious and often untimely. A centralized database and a web-based portal that integrate information derived by different types of slope monitoring systems has been developed. The paper illustrates the specific features of the developed "SensorNet" and provides examples of its use for visualizing and analyzing in an integrated manner data from different monitoring systems. In perspective it could serve as an every-day operational tool for a timely reporting of landslide monitoring data for surveillance and warning purposes.
\end{abstract}

\section{INTRODUCTION}

Monitoring weather-induced landslides is of significant importance for risk management in Emilia Romagna Region, since they are often the cause of civil protection emergencies (Bertolini et al. 2005). Technical Basin Services are the regional institutions that carry out most of the landslide monitoring activity (in some cases in collaboration with Universities) that can be used to study the characteristics of phenomena (Corsini et al. 2005), to operate evacuation plans plan and to design structural countermeasures (Corsini et al. 2006).

The use of multi-sensors integrated system for landslide monitoring poses some critical issues in system setup and data management (Bertacchini et al. 2013). Above all, the use of different noninteroperable systems and the fragmentation of datasets in local data repositories limit the timely availability and spreading of data among end users such as Civil Protection, Municipalities and Technical Basin Services.

The objective of the research was to develop and implement a centralized database and a web-based data management center in order to integrate data from different sensors relevant to landslide monitoring. The aim is to give equal data access possibility to all the institutional endusers. The idea is to create a data portal similar in principle - but different in technology - to these already available in some other Italian regions, such as for instance Lombardia (see for example ARPA Lombardia-Centro Monitoraggio Geologico. 2015).

The development of a landslide monitoring data portal (SensorNet) for the Emilia Romagna Region, was carried out by Lepida SpA, a public owned company that provides data warehousing and services to the public administrations. Technical Basin Services of Regione Emilia Romagna and the University of Modena and Reggio Emilia supported this activity by providing data and by defining the specific functions that had to be implemented in the portal in order to interrogate and visualize data in formats suitable for institutional end-users.

The paper illustrates the specific features of SensorNet and provides examples of its usage for visualizing data from different monitoring systems. SensorNet has reached in 2015 a first level of operational development and it now integrates data from different types of monitoring systems deployed on landslides in the Emilia Romagna Apennines. 


\section{CHARACTERISTICS OF CENTRALIZED DATA MANAGEMENT CENTER (SENSORNET)}

\subsection{General issues and requisites}

Several landslide monitoring systems are used in Emilia Romagna by public services at the regional to the municipality level. However, monitoring systems are generally non-interoperable and they are commonly provided by a variety of private suppliers. This results in a multiplicity of databases managed by different entities. This multiplication of management systems makes datasets unsafe and it generates difficulties in sharing and integrating data. Moreover, the large use of operator-based standalone systems determines unnecessary loads of work for calculating data derivatives.

The integration of data from different monitoring systems into a single centralized data management center can therefore facilitate the availability of data and improve related services in the short and long-term. It can also boost the capacity of making data available and shared among endusers and can lead to optimization of economic resources allocation.

The "SensorNet" platform was developed to meet the following requisites: to be a management and access interface for different non-interoperable monitoring systems; to be able to integrate data from both automated and non-automated monitoring sensors; to be web-based so to allow data access from anywhere; to provide customized access to data to users; to be an added value "internet of things" application based upon already existing services.

\subsection{Type of monitoring data and data flows}

As of now, landslide monitoring data integrated in SensorNet derive from: (i) systems operated in fully automated mode (such as total stations, crack meters, in-place inclinometers, pressure transducers equipped with data-loggers and data transmission units); (ii) systems operated in non-automated mode (such as, for instance, inclinometer casings, load cells with standalone data loggers). SensorNet also integrates data from the fully automated regional hydro-meteorological monitoring network of ARPA Emilia Romagna (rain-gauges and stream-gauges). Landslide monitoring data, as well as hydro-meteorological data, can be accessed from the mainframe of SensorNet.

The information flow from landslide monitoring systems operated in fully automated mode foresees the presence of a middleware that uniforms the different proprietary data formats that are sent by the data loggers and transmission units (GPRS and Tetra radio modems) (Fig. 1a).
As regards topographic data from total stations, they are managed and corrected in a proprietary application at University of Modena. Validated data are then included in a SQL database in the unimore server from where they are directly captured by SensorNet.

The information flow from landslide monitoring systems operated in non-fully automated mode requires the operators to export the data in a pre-defined standardized format and to load such files in an ftp site (Fig. 1b). From the ftp site, the SensorNet retrieves the most updated files and includes the data in the server database.

For both fully automated and non-fully automated systems, the SensorNet system calculates derivative measures and allows data management and visualization, multi-sensor plotting and data download.

More specifically, predefined multi-sensor plots are implemented in order to make data readily available for pre-defined relevant group of slope monitoring sensor and compare displacement patterns to rainfall recorded in the ARPA network. This is important in landslide sites that require periodic data

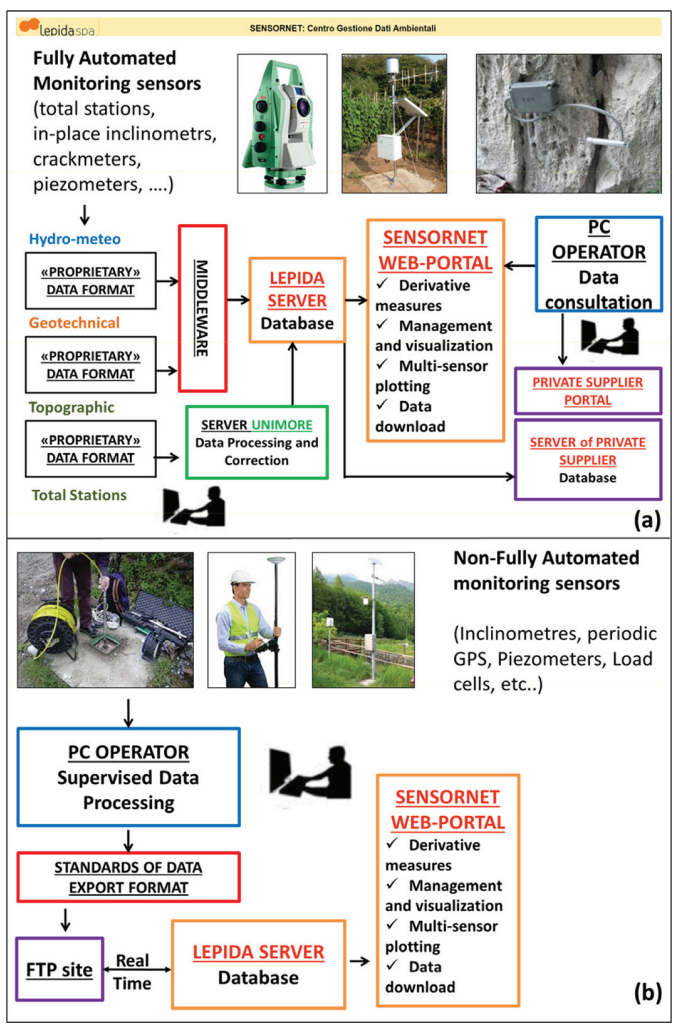

Figure 1. (a) Scheme of data flow for fully automated sensors; (b) Scheme of data flow for non-fully automated sensors. 
reporting and real time data update in order to manage evacuation plans or to assure close surveillance.

\section{EXAMPLES OF APPLICATION}

\subsection{Automated systems: Total station monitoring}

The case study of the Capriglio landslide (province of Parma) is presented as an example of integration in SensorNet of data from a fully automated total station (Fig. 2a). The total station is operated on the site since soon after the landslide reactivation event of 2013. Displacement data from this monitoring system are used as warning thresholds within the evacuation plan defined by the regional civil protection agency and the local municipality.

In order to provide significant data, SensorNet calculates derivatives of the original measures. Specifically, it derives the displacement vector by combining in real time the inclined distance from ATS to reflectors and the zenith and horizontal angles measured by the total stations (that operate with polar surveys). The availability of the displacement vector is particularly valuable in situations in which the direction of movement is close to the orthogonal to the line of sight of the ATS beam (such as in the group of prisms labelled as part of group "Pianestolla" represented in Figure 2a). On
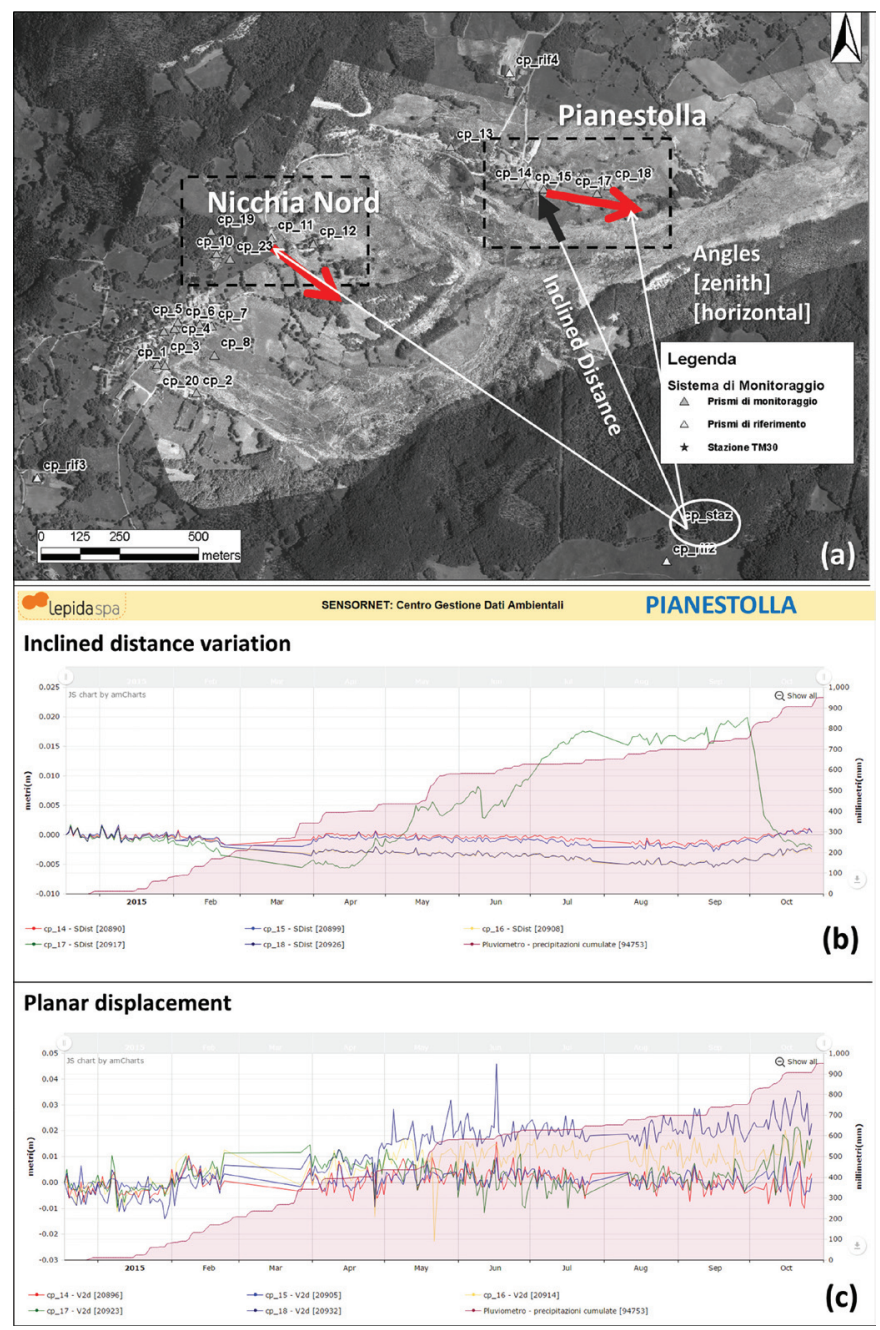

Figure 2. (a) Ortho-photo of landslide site monitored by Automated Total Station (ATS) with indication of slope displacement directions; (b) Example of inclined distance variation plot; (c) Example of inclined distance variation plot. 
the other hand, it is less important if movements occurs in a direction coincident to the line of sight (such as in the group of prisms labelled as part of group "Nicchia Nord" represented in Figure 2a).

In SensorNet, the user can select pre-defined plots for groups of prisms that have been defined as relevant. For each group, the operator can then choose to visualize the inclined distance variation plot (Fig. 2b) or the planar displacement plot (Fig. 2c). The first plot is generally less "noisy" in terms of data variability, but it might provide an of displacement. The second one might be more "noisy", as it derives from the combination of different data affected by some levels of errors, but it does provide a more realistic assessment of true displacement. On the other hand, as beforehand mentioned, this difference is not so great in case of movements directed along the ATS-prism line of sight, so the user might in these cases prefer to visualize the less noisy dataset of inclined distance variation.

Predefined plots are updated in near-real time (i.e. immediately after the cycle of measurements has been completed, transferred to the unimore server for automated correction and included in the SQL server). These plots are also displayed with a nearreal time updated value of cumulated rainfall that is retrieved from the selected representative rain gauge of the ARPA monitoring network. In the plot window, zoom-in functions are provided to the user, that can select a specific time window to be displayed. Moreover, these plots can be customized by selecting the date of a virtual "zero" (so to highlight the displacement pattern from a specific date onward). Another customization function allows to enabledisable a specific sensor (i.e. prism) from the plot.

\subsection{Non-Automated systems: periodic measurements of inclinometer casings}

Several hundreds of inclinometer casings have been installed in the last decades in tens of different landslides across the Apennines (Bertolini et al., 2005). Out of these, only a dozen has been fully automated by installing in-place inclinometers and GPRS/Tetra transmission modems.

This implies that there is a huge amount of monitoring data from periodic surveys (carried out with portable inclinometer measurement systems) that are stored in various formats in different offices. This makes their timely consultation difficult.

The solution chosen to integrate data from period inclinometric surveys in SensorNet, was to export the available survey data (i.e. the tilt values in the A and $B$ axes measured during each survey) in a standardized pre-defined txt format and to upload these files in a .ftp site from where the most updated measurement file of a given inclinometer is automatically retrieved and it is added up to other files referring

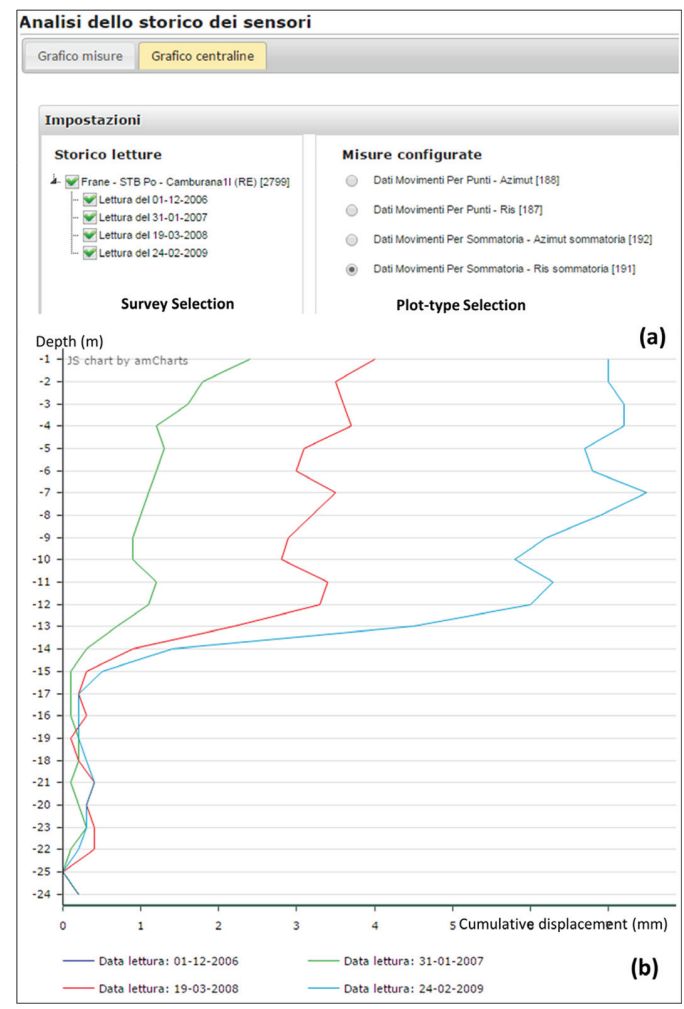

Figure 3. (a) Inclinometric survey and plot-type selection window; (b) Cumulative displacement plot in SensorNet.

to the same inclinometer. The survey data are then post-processed in SensorNet in order to obtain derivative measures that enables the data management systems to elaborate inclinometer plots.

The list of inclinometers that can be interrogated is displayed in a selection tree in the "Sensors" section. Once a specific inclinometer is selected, a surveys selection and a plot-type selection frames are activated (Fig. 3a). This allows any of the surveys stored in the database to be selected and to proceed with the visualization of the following alternative plots: cumulative displacement (see Fig. 3b), incremental displacement, azimuth of displacement (both for the incremental and cumulative) (Dunnicliff \& Green 1988).

\section{DISCUSSION}

The completion of the database is requires personnel to take care of the collection, homogenization in the required formats and upload to the server "historic" data from non-fully automated monitoring systems (e.g. inclinometers) and automated systems 
connected to other databases. Moreover, it requires linking to the database all the already existing data loggers serving automated monitoring systems. To do so, it is crucial to obtain the specific coding formats and to implement middleware conversion tools.

The further implementation of the database requires, first of all, trained staff dedicated to field data acquisition for non-automated monitoring systems, data validation and data transfer. The installation of automated monitoring systems and conversion into automated mode of the systems that are considered strategic are also important. For example, sensors used to control the functionality of slope stabilization works or these used for emergency plans are certainly to be automatized. The standardization of output data format from data loggers installed by suppliers is also necessary. An obligation on data loggers providers to direct data to the central data management center should be introduced. Maintenance of monitoring systems is also a crucial issue, together with close control of the operational status, replacement of sensors, updating software, etc. Hiring monitoring systems for certain periods could be a mean to overcome maintenance problems. The definition of new simplified procedures for the provision of SIM cards to public offices to be used for data transmission via GPRS or provision of TETRA radio modems could also be of help.

The further development of the SensorNet portal should include multi sensor interrogation functionalities in a georeferenced mapping framework, in which thematic maps available via "wms" services (topographic, geologic, etc..) should be included. Specific plugins for advanced data analysis based on statistic and/or artificial intelligence algorithms could also be an innovative option for making data more usable by non-experts. This should pair with the development of metadata to facilitate the interpretation of data by inexperienced operators (e.g. municipal technical offices that have to manage evacuation plans), a tack that links to the foreseen spread to new potential users (local authorities and professional consultants).

\section{CONCLUSIONS}

The paper illustrates some of the specific features of SensorNet, a platform for multi sensor data integration at regional scale. The paper provides some examples of its use for visualizing and analyzing in an integrated manner data from different monitoring systems. SensorNet has reached a first operational stage of development.

In perspective, it could be further developed and serve as an every-day operational tool for a timely reporting of landslide monitoring data for surveil- lance and warning purposes. To such aim, the key issues that need to be solved are: (i) the long term availability of personnel and structures specifically dedicated to the acquisition, transmission and validation of data by the various monitoring systems (i) the assessment of a long-term funding scheme that can assure the continuity of maintenance of monitoring systems; (iii) the clear definition of specific responsibilities for validation, interpretation and use of data from which to derive geological models, assess trigger thresholds, activate attention and alarm phases based on monitoring data.

With respect to these issues, the operational prototype of SensorNet represents the demonstration of the technical feasibility of a useful "internet of things" application. Hopefully, it will be a stimulus for discussion of these key issues in the appropriate institutional structures.

\section{ACKNOWLEDGEMENTS}

This work was supported by the Civil Protection Agency of the Emilia-Romagna Region under the framework agreement "Special activities on support to the forecast and emergency planning of Civil Protection with respect to hydrogeological risk" (ASPER-RER, 2011-2015).

\section{REFERENCES}

ARPA Lombardia-Centro Monitoraggio Geologico. 2015. http://cmg.arpalombardia.it/webcmgfrontend/ ilcentro.asp.

Bertacchini, E. Castagnetti, C. Corsini, A. Capra, A. 2013. Multi-sensors integrated system for landslide monitoring: critical issues in system setup and data management. European Journal of Remote Sensing, 46, 104-124.

Bertolini, G. Guida, M. Pizziolo, M. 2005. Landslides in EmiliaRomagna region (Italy): strategies for hazard assessment and risk management. Landslides 2(4), 302-312.

Corsini, A. Borgatti, L. Pellegrini, M. Ronchetti, F. Borghi, A. Campagnoli, I. Caputo, G. Gatti, A. Leuratti, E. Lucente, C.C. Truffelli, G. Sartini, G. 2005. Monitoraggio di grandi frane riattivate e sospese nella valle del Fiume Secchia (Appennino settentrionale). Giornale di Geologia Applicata, 2, 35-44.

Corsini, A. Borgatti, L. Caputo, G. De Simone, N. Sartini, G. Truffelli, G. 2006. Investigation and monitoring on support to the structural mitigation of large slow moving landslides: an example from Ca' Lita (Northern Apennines, Reggio Emilia, Italy). Natural Hazards and Earth System Sciences, 6, 55-61.

Dunnicliff, J. \& Green, G.E 1988. Geotechnical instrumentation for monitoring field performance. WileyInterscience Publication, 577 pp. ISBN 0-471-00546-0. 